\title{
Adaptive Co-primary Shared Access Between Co-located Radio Access Networks
}

\author{
Sofonias Hailu, Alexis A. Dowhuszko and Olav Tirkkonen \\ Department of Communications and Networking, Aalto University, P.O. Box 13000, FI-00076 Aalto, Finland \\ Email: \{sofonias.hailu, alexis.dowhuszko, olav.tirkkonen\}@aalto.fi
}

\begin{abstract}
Inter-operator spectrum sharing is expected to play a key role in fulfilling the predicted spectrum demand for future wireless systems. In this work, we propose an adaptive co - primary shared access scheme between co-located Radio Access Networks (RANs) owned by different operators. In the literature, the main focus is on orthogonal spectrum sharing. In this work, the spectrum is adaptively partitioned into private and non-orthogonally shared frequency sub-bands with the aim of maximizing inter-RAN sum rate. The non-orthogonally shared frequency sub-band is simultaneously used by all the RANs, with inter-RAN interference minimized. A zero-forcing precoder is used to serve the users scheduled in the private frequency subband. With the exchange of inter-RAN channel state information, a sparse precoder is used as inter-RAN precoder to serve the users scheduled in the shared frequency sub-band. Using a heuristic algorithm based on user grouping, spectrum partitioning and user scheduling are optimized. Based on simulation results, the proposed adaptive spectrum sharing approach outperforms conventional approaches of orthogonal and full-band non-orthogonal spectrum sharing.
\end{abstract}

\section{INTRODUCTION}

Spectrum demand predictions for future wireless networks indicate that new inter-operator spectrum sharing approaches need to be introduced in order to fulfill the expected requirement. In [1], a 10-fold increase in spectrum is predicted by 2020 when compared to 2010 , in order to fulfill the exponentially growing data demand. It is expected that a bandwidth of $1200-1700 \mathrm{MHz}$ will be required by 2020 to fulfill the mobile data demand [2].

Spectrum can be shared orthogonally or nonorthogonally [3]. In orthogonal spectrum sharing, multiple operators are prevented from simultaneously using the same frequency resource. Game theoretic approaches for orthogonal spectrum sharing have been discussed in the literature, see e.g. [4]. In non-orthogonal spectrum sharing, on the other hand, multiple operators are allowed to simultaneously use the same frequency resource. Therefore, inter-operator interference is generated in this situation, and the control of this impairment becomes challenging. In [3], [5], different transmit beamforming techniques are applied to cope with the inter-operator interference. In [6], non-orthogonal spectrum sharing is modeled as a strategic non-cooperative game, and game theoretical distributed algorithms are proposed. However, such aggressive full-band spectrum sharing approaches lead only to marginal overall gains [7].

Recently, the concept of co-primary shared access was introduced [9]. The main idea of the co-primary shared access model is that primary license holders agree to jointly use (part of) their spectrum. In practice, only users located close to their base station benefit when non-orthogonal spectrum sharing is used [8]. For this reason, a fractional spectrum sharing scheme was investigated in [8], where the available spectrum is partitioned into orthogonally and non-orthogonally shared portions. In addition, interference alignment is used to remove the inter-operator interference, at the expense of reducing the spatial degrees of freedom. In [8], the partitioning of the spectrum and the maximum distance between nonorthogonally sharing users and the the serving base station are determined statically.

In this paper, we propose a co-primary shared access scheme where the spectrum is adaptively partitioned to orthogonally and non-orthogonally shared frequency sub-bands. The adaptation of the partitioning is performed at the scheduling interval level, taking the channel conditions of the users into account. We consider a scenario where the operators apply joint multipoint transmission within their own Radio Access Network (RAN), and coordinate their transmissions to minimize interoperator interference. A sparse precoder [10] is applied as the inter-RAN precoder to minimize inter-operator interference. As a rule of thumb, a user is served in the non-orthogonally shared frequency sub-band if the combined effect of the signal degradation (due to inter-operator interference) and the resulting frequency reuse gain becomes beneficial. Substantial gain is shown when using the proposed inter-RAN adaptive co-primary shared access scheme.

The rest of the paper is organized as follows: Section II presents the system model, while Section III presents sparse precoder and discusses how it is applied as inter-operator precoder. In Section IV, the optimization problem is formulated and the proposed algorithm for adaptive spectrum partitioning is described. Simulation results are presented and analyzed in Section V. Finally, conclusions are drawn in Section VI.

\section{SYSTEM MODEL}

\section{A. Multi-RAN Joint Transmission Channel}

We consider a downlink transmission system consisting of two co-located RANs owned by two different mobile network operators. The RAN $r \in\{1,2\}$ is composed of $N_{r}$ Transmitters (TXs) and $N_{r}$ Receivers (RXs). For simplicity we consider the case where the TXs and RXs are equipped with single-element antennas. We assume that each RAN has an equal amount of licensed spectrum, and they are willing 
to adaptively share part of their spectrum. The spectrum is partitioned such that each RAN has an equal-sized private part of the spectrum, while the remaining part is non-orthogonally shared between the RANs. The two RANs share signalling and inter-RAN global Channel State Information (CSI) for efficient spectrum sharing. However, inter-RAN user data sharing is not allowed due to both privacy reasons and backhaul requirement limitations.

Within the private resources, the RANs use zero-forcing precoders to jointly serve the RXs scheduled in the private frequency sub-band. The RXs scheduled in the shared frequency sub-band are served using a sparse precoder, which mitigates inter-RAN interference. Without loss of generality, both precoders use an equal per-stream power constraint.

Wireless channels between TXs and RXs are modeled combining the effect of distance dependent pathloss and flat Rayleigh fading of unitary variance. Noise is modeled as zero mean i.i.d. complex circularly symmetric Gaussian random variable of noise power $\sigma^{2}$.

For simplicity, a user of an operator may get communication resources either in the private or the shared part of the spectrum, but not in both parts simultaneously. Accordingly, we denote the set of RXs scheduled in the private frequency sub-band of RANs 1 and 2 by $\mathcal{U}_{\mathrm{p}, 1}$ and $\mathcal{U}_{\mathrm{p}, 2}$, respectively, and the set of RXs scheduled in the shared frequency sub-band of both operators as $\mathcal{U}_{\mathrm{s}, 1}$ and $\mathcal{U}_{\mathrm{s}, 2}$. Furthermore, let us denote the number of RXs scheduled in the private and shared frequency sub-bands of RANs 1 and 2 as $N_{\mathrm{p}, 1}=\left|\mathcal{U}_{\mathrm{p}, 1}\right|, N_{\mathrm{p}, 2}=\left|\mathcal{U}_{\mathrm{p}, 2}\right|$, $N_{\mathrm{s}, 1}=\left|\mathcal{U}_{\mathrm{s}, 1}\right|$ and $N_{\mathrm{s}, 2}=\left|\mathcal{U}_{\mathrm{s}, 2}\right|$.

The received signal vector of the RXs scheduled in the private frequency sub-band of RAN $r$ is

$$
\mathbf{y}^{(\mathrm{p}, r)}=\mathbf{H}^{(\mathrm{p}, r)} \mathbf{W}^{(\mathrm{p}, r)} \mathbf{s}^{(\mathrm{p}, r)}+\boldsymbol{\eta}^{(\mathrm{p}, r)},
$$

where the elements of $\mathbf{y}^{(\mathrm{p}, r)} \in \mathbb{C}^{N_{\mathrm{p}, r} \times 1}$ are signals $y_{i}^{(\mathrm{p}, r)}$ which are received at the $i$-th $\mathrm{RX}$ in the private frequency sub-band of RAN $r$. The intra-RAN channel matrix is $\mathbf{H}^{(\mathrm{p}, r)}=\left[\mathbf{h}_{1}^{T} \ldots \mathbf{h}_{N_{\mathrm{p}, r}}^{T}\right]^{T} \in \mathbb{C}^{N_{\mathrm{p}, r} \times N_{r}}$, where element $h_{i, j}^{(\mathrm{p}, r)}$ is the channel gain from TX $j$ in RAN $r$ to RX $i \stackrel{i}{\in} \mathcal{U}_{\mathrm{p}, r}$. The intra-RAN precoder matrix is represented by $\mathbf{W}^{(\mathrm{p}, r)}=\left[\mathbf{w}_{1} \ldots \mathbf{w}_{N_{\mathrm{p}, r}}\right] \in \mathbb{C}^{N_{r} \times N_{\mathrm{p}, r}}$ where $w_{j, i}^{(\mathrm{p}, r)}$ is the beamforming vector for transmitting the data symbols to RX $i \in \mathcal{U}_{\mathrm{p}, r}$ from TX $j$ of RAN $r$. The $N_{\mathrm{p}, r} \times 1$ vector $\mathbf{s}^{(\mathrm{p}, r)}$ contains the data symbols for the RXs in $\mathcal{U}_{\mathrm{p}, r}$, where $\mathbf{s}_{i}^{(\mathrm{p}, r)} \in \mathcal{C N}(0,1)$. Finally, the vector $\boldsymbol{\eta}^{(\mathrm{p}, r)} \in \mathbb{C}^{N_{\mathrm{p}, r} \times 1}$ contains the external interference plus noise power at the RXs in $\mathcal{U}_{\mathrm{p}, r}$.

The beamforming vector $j$ used on the private part of the frequency is based on zero forcing with equal per-stream power. Let

$$
\mathbf{T}=\left[\mathbf{t}_{1} \ldots \mathbf{t}_{N_{\mathrm{p}, r}}\right]=\left(\mathbf{H}^{(\mathrm{p}, r)} \mathbf{H}^{(\mathrm{p}, r)^{\mathrm{H}}}\right)^{-1} .
$$

The beamforming vector used for the transmission of the data symbol to $\mathrm{RX} j$ is

$$
\mathbf{w}_{j}=\sqrt{p} \frac{\mathbf{H}^{(\mathrm{p}, r)^{\mathrm{H}}} \mathbf{t}_{j}}{\left\|\mathbf{H}^{(\mathrm{p}, r)^{\mathrm{H}}} \mathbf{t}_{j}\right\|_{2}},
$$

where $(\cdot)^{\mathrm{H}}$ and $(\cdot)^{-1}$ denote the Hermitian and inverse of the corresponding matrix, respectively. Note that the normalization factor that appears in (3) is due to the equal per-stream power constraint $\left(\left\|\mathbf{w}_{j}\right\|^{2}=P, P>0\right)$.

The RXs scheduled on the shared frequency sub-band are combined in one signal model for both operators,

$$
\mathbf{y}^{(\mathrm{s})}=\mathbf{H}^{(\mathrm{s})} \mathbf{W}^{(\mathrm{s})} \mathbf{s}^{(\mathrm{s})}+\boldsymbol{\eta}^{(\mathrm{s})} .
$$

Here, $\mathbf{y}^{(\mathrm{s})} \in \mathbb{C}^{N_{\mathrm{s}} \times 1}$ is the received signal vector, where $N_{\mathrm{s}}=N_{\mathrm{s}, 1}+N_{\mathrm{s}, 2}$. The inter-RAN channel matrix is denoted as $\mathbf{H}^{(\mathrm{s})}=\left[\mathbf{h}_{1}^{T} \ldots \mathbf{h}_{N_{\mathrm{s}}}^{T}\right]^{T} \in \mathbb{C}^{N_{\mathrm{s}} \times N}$ and the inter-RAN precoder matrix as $\mathbf{W}^{(\mathrm{s})}=\left[\mathbf{w}_{1}, \ldots, \mathbf{w}_{N_{\mathrm{s}}}\right] \in \mathbb{C}^{N \times N_{\mathrm{s}}}$, where $N=N_{1}+N_{2}$ is the total number of TXs in both RANs. The $N_{\mathrm{s}} \times 1$ vectors $\mathbf{s}^{(\mathrm{s})}$ and $\boldsymbol{\eta}^{(\mathrm{s})}$ denote the data symbol, and external interference and noise power vectors, respectively.

The sparse inter-RAN precoder which is used on the shared part of the spectrum will be discussed in Section III.

\section{B. System Performance Model}

For simplicity, we consider the inter-RAN sum rate of the users as a perfromance metric. Let us denote the total amount of spectrum that is available at both operators as $B$. During a scheduling interval, let us assume that bandwidth $B_{\mathrm{s}}$ is nonorthogonally shared between both RANs, and the rest of the bandwidth is equally divided between the RANs in such a way that each RAN ends having private bandwidth $B_{\mathrm{p}}$. The rate of a user $i \in \mathcal{U}_{\mathrm{p}, r}$ scheduled in the private frequency sub-band of RAN $r$ is

$$
R_{\mathrm{p}, i}=B_{\mathrm{p}} \log _{2}\left(1+\frac{\left|\mathbf{h}_{i}^{T} \mathbf{w}_{i}\right|^{2}}{\eta_{i}}\right),
$$

while the rate of a user $i \in \mathcal{U}_{\mathrm{s}, r}$ scheduled in the shared frequency sub-band is

$$
R_{\mathrm{s}, i}=B_{\mathrm{s}} \log _{2}\left(1+\frac{\left|\mathbf{h}_{i}^{T} \mathbf{w}_{i}\right|^{2}}{\eta_{i}+\sum_{j \neq i}\left|\mathbf{h}_{i}^{T} \mathbf{w}_{j}\right|^{2}}\right) .
$$

Note that the summation term in the denominator of (6) is due to the inter-RAN interference, while it is zero in (5) due to the use of intra-RAN joint transmission.

\section{SPARSE PRECODING FOR INTER-RAN PRECODER}

We use sparse precoder as inter-RAN precoder. In [10], sparse precoder was proposed to optimize data symbol routing to TXs in a multi-cell environment with a constraint on the total amount of data symbols that could be shared. For completeness, in this section we briefly review how sparse precoder works and the way in which it can be applied as inter-RAN precoder.

\section{A. Routing Matrix}

A routing matrix determines the way in which the data symbols for the RXs are routed through the different TXs. Since data symbol is not shared between the RANs, the routing matrix $\mathbf{D}=\left[\mathbf{d}_{1} \ldots \mathbf{d}_{N_{\mathrm{s}}}\right] \in\{0,1\}^{N \times N_{\mathrm{s}}}$ for determining the 
shape of the inter-RAN precoder $\mathbf{W}^{(\mathrm{s})}$, i.e. $\mathbf{W}^{(\mathrm{s})}=\mathbf{W}^{(\mathrm{s})} \odot \mathbf{D}$, is defined as

$$
d_{i, j}= \begin{cases}1 & \text { if } T X i \in \mathcal{O}^{(k)}, R X j \in \mathcal{U}_{\mathrm{s}, r}, k=r \\ 0 & \text { otherwise }\end{cases}
$$

where the sets $\mathcal{O}^{(1)}$ and $\mathcal{O}^{(2)}$ denote the sets of the TXs of RANs 1 and 2, respectively. The symbol ' $\odot$ ' denotes an element-wise multiplication between two matrices or vectors.

\section{B. Inter-RAN Precoder for a Given Routing Matrix}

With zero-forcing criterion, the maximization of interRAN sum rate can be replaced by the minimization of the emitted inter-RAN interference, i.e. the Frobenius norm $\left\|\mathbf{H}^{(\mathrm{s})}\left(\mathbf{W}^{(\mathrm{s})} \odot \mathbf{D}\right)-\mathbf{I}\right\|_{\mathrm{F}}$, where $\mathbf{I}=\left[\mathbf{e}_{1} \ldots \mathbf{e}_{N_{\mathrm{s}}}\right]$ is an identity matrix of size $N_{\mathrm{s}}$. The element-wise multiplication between $\mathbf{W}^{(\mathrm{s})}$ and $\mathbf{D}$ is included to force the inter-RAN precoder bear the shape of the routing matrix $D$. Due to the per-stream power constraint and the relationship

$$
\left\|\mathbf{H}^{(\mathrm{s})}\left(\mathbf{W}^{(\mathrm{s})} \odot \mathbf{D}\right)-\mathbf{I}\right\|_{\mathrm{F}}^{2}=\sum_{j \in \mathcal{U}_{\mathrm{s}, 1} \cup \mathcal{U}_{\mathrm{s}, 2}}\left\|\mathbf{H}^{(\mathrm{s})}\left(\mathbf{w}_{j} \odot \mathbf{d}_{j}\right)-\mathbf{e}_{j}\right\|_{2}^{2},
$$

the beamforming vectors of $\mathbf{W}^{(\mathrm{s})}$ can be computed independently [10], [11]. Therefore, the $j$-th column of $\mathbf{W}^{(\mathrm{s})}$ can be computed from the following linear least-squares minimization problem

$$
\underset{\mathbf{w}_{j}}{\operatorname{minimize}}\left\|\mathbf{H}^{(\mathrm{s})}\left(\mathbf{w}_{j} \odot \mathbf{d}_{j}\right)-\mathbf{e}_{j}\right\|_{2}^{2} .
$$

The computational complexity of (9) can be reduced since the beamforming vector $\mathbf{w}_{j}$ has a sparse structure. Let us denote the set of non-zero entries of the beamforming vector $\mathbf{w}_{j}$ as $\mathcal{I}=\left\{i \mid w_{i, j} \odot d_{i, j} \neq 0\right\}$, and the set of the nonidentically zero rows of the inter-RAN channel matrix as $\mathcal{J}=\left\{j \mid \mathbf{h}_{j}^{T}(\mathcal{I}) \neq \mathbf{0}_{1 \times|\mathcal{I}|}\right\}$. The set $\mathcal{J}$ can be non-empty, e.g. if weak channel gains are approximated as zeros and the RANs exchange only a limited amount of CSI information. Equation (9) can be re-written as follows:

$$
\underset{\mathbf{w}_{j}(\mathcal{I})}{\operatorname{minimize}}\left\|\mathbf{H}^{(\mathrm{s})}(\mathcal{I}, \mathcal{J})\left(\mathbf{w}_{j}(\mathcal{I}) \odot \mathbf{d}_{j}(\mathcal{I})\right)-\mathbf{e}_{j}(\mathcal{I})\right\|_{2}^{2} .
$$

One way to solve the linear least-squares minimization problem is based on using QR-decomposition. Let $\lambda_{1}=|\mathcal{I}|$ and $\lambda_{2}=|\mathcal{J}|$. Then the $\mathrm{QR}$-decomposition of the reduced channel matrix can be written as

$$
\mathbf{H}^{(\mathrm{s})}(\mathcal{I}, \mathcal{J})=\mathbf{Q}\left[\begin{array}{c}
\mathbf{R} \\
\mathbf{0}_{\left(\lambda_{1}-\lambda_{2}\right) \times \lambda_{2}}
\end{array}\right],
$$

where $\mathbf{Q} \in \mathbb{C}^{\lambda_{1} \times \lambda_{1}}$ is an orthogonal matrix, and $\mathbf{R} \in \mathbb{C}^{\lambda_{2} \times \lambda_{2}}$ is an upper triangular matrix. Finally, the solution of the optimization problem (10), including the per-stream power constraint, is given by [10]:

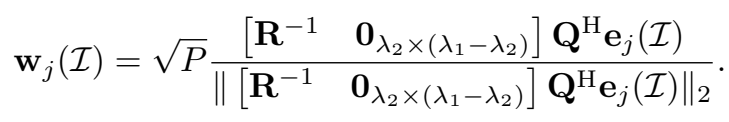

\section{ADAPTIVE SPECTRUM PARTITIONING}

\section{A. Formulation of the Optimization Problem}

The main objective of this work is to maximize the inter-RAN sum rate by means of optimizing the adaptive partitioning of the spectrum into private and shared frequency sub-bands and the scheduling of the RXs into both sub-bands. Let us denote the set of user grouping by $\mathcal{U}=\left\{\mathcal{U}_{\mathrm{p}, 1}, \mathcal{U}_{\mathrm{p}, 2}, \mathcal{U}_{\mathrm{s}, 1}, \mathcal{U}_{\mathrm{s}, 2}\right\}$. The spectrum partitioning can be uniquely determined by $B_{\mathrm{s}}$ where $2 B_{\mathrm{p}}+B_{\mathrm{s}}=B$. Then, the optimization problem becomes

$$
\begin{array}{cl}
\underset{\mathcal{U}, B_{\mathrm{s}}}{\operatorname{maximize}} & \sum_{r=1}^{2} \sum_{i \in \mathcal{U}_{\mathrm{p}, r}} R_{\mathrm{p}, i}+\sum_{r=1}^{2} \sum_{j \in \mathcal{U}_{\mathrm{s}, r}} R_{\mathrm{s}, j} \\
\text { subject to } & \mathcal{U}_{\mathrm{p}, 1} \cap \mathcal{U}_{\mathrm{p}, 2} \cap \mathcal{U}_{\mathrm{s}, 1} \cap \mathcal{U}_{\mathrm{s}, 2}=\emptyset \\
& 0 \leq B_{\mathrm{s}} \leq B, 2 B_{\mathrm{p}}+B_{\mathrm{s}}=B \\
& \left\|\mathbf{w}_{j}\right\|^{2}=B_{\mathrm{p}} P, \quad \forall j \in \mathcal{U}_{\mathrm{p}, 1} \cup \mathcal{U}_{\mathrm{p}, 2} \\
& \left\|\mathbf{w}_{j}\right\|^{2}=B_{\mathrm{s}} P, \quad \forall j \in \mathcal{U}_{\mathrm{s}, 1} \cup \mathcal{U}_{\mathrm{s}, 2}
\end{array}
$$

where the first constraint indicates that a given RX is scheduled either in the private or shared frequency sub-band, while the second constraint shows the possible ways of partitioning the spectrum. The last two constraints indicate that equal perstream power spectral density is applied in order to distribute transmission power evenly over the whole spectrum, and to avoid strong interference in some frequency sub-bands of the spectrum. For a given spectrum partition $B_{\mathrm{s}}$ and user grouping $\mathcal{U}$, the rates in the objective function are calculated using (5) and (6), while the intra-RAN and inter-RAN precoders are obtained according to (3) and (12), respectively.

\section{B. Heuristic Algorithm based on User Grouping}

In the optimization problem, we need to maximize the interRAN sum rate over $\mathcal{U}$ and $B_{\mathrm{s}}$. For a fixed $B_{\mathrm{s}}$, the maximization of the inter-RAN sum rate over $\mathcal{U}$ is a combinatorial problem. If we assume that the spectrum is infinitely divisible, the spectrum can be partitioned in infinite ways. However, the inter-RAN sum rate for a fixed $\mathcal{U}$ is a linear function of $B_{\mathrm{s}}$. Therefore, with a given user grouping criterion for a fixed $B_{\mathrm{s}}$, the function that describes inter-RAN sum rate with respect to $B_{\mathrm{s}}$ has discontinuities at values of $B_{\mathrm{s}}$ where $\mathcal{U}$ changes.

In the proposed algorithm, we search for the maximum inter-RAN sum rate over the left and right limits of the discontinuity points of the objective function over $B_{\mathrm{s}}$ and $\mathcal{U}$, and the inter-RAN sum rates at $B_{\mathrm{s}}=0$ and $B_{\mathrm{s}}=B$. The discontinuity points are iteratively computed taking the updated values of $\mathcal{U}$ and $B_{\mathrm{s}}$ in each iteration. The set $\mathcal{U}$ and the value of $B_{\mathrm{s}}$ are updated using the spectral efficiencies that an $\mathrm{RX} i \in \mathcal{U}_{\mathrm{p}, r}$ would achieve in the private and shared frequency sub-bands, respectively denoted by $S_{\mathrm{p}, i}$ and $S_{\mathrm{s}, i}$, and the scheduling criterion. The $S_{\mathrm{s}, i}$ is the spectral efficiency that the RX $i \in \mathcal{U}_{\mathrm{p}, r}$ would achieve if $\mathcal{U}_{\mathrm{p}, r} \leftarrow \mathcal{U}_{\mathrm{p}, r} \backslash\{i\}$ and $\mathcal{U}_{\mathrm{s}, r} \leftarrow \mathcal{U}_{\mathrm{s}, r} \cup\{i\}$. The RANs exchange beamforming information of the RXs in $\mathcal{U}_{\mathrm{s}, r}$ in order to estimate the interRAN interference. For fairness, we assume that each RAN applies a scheduling criterion where a RX is scheduled in 


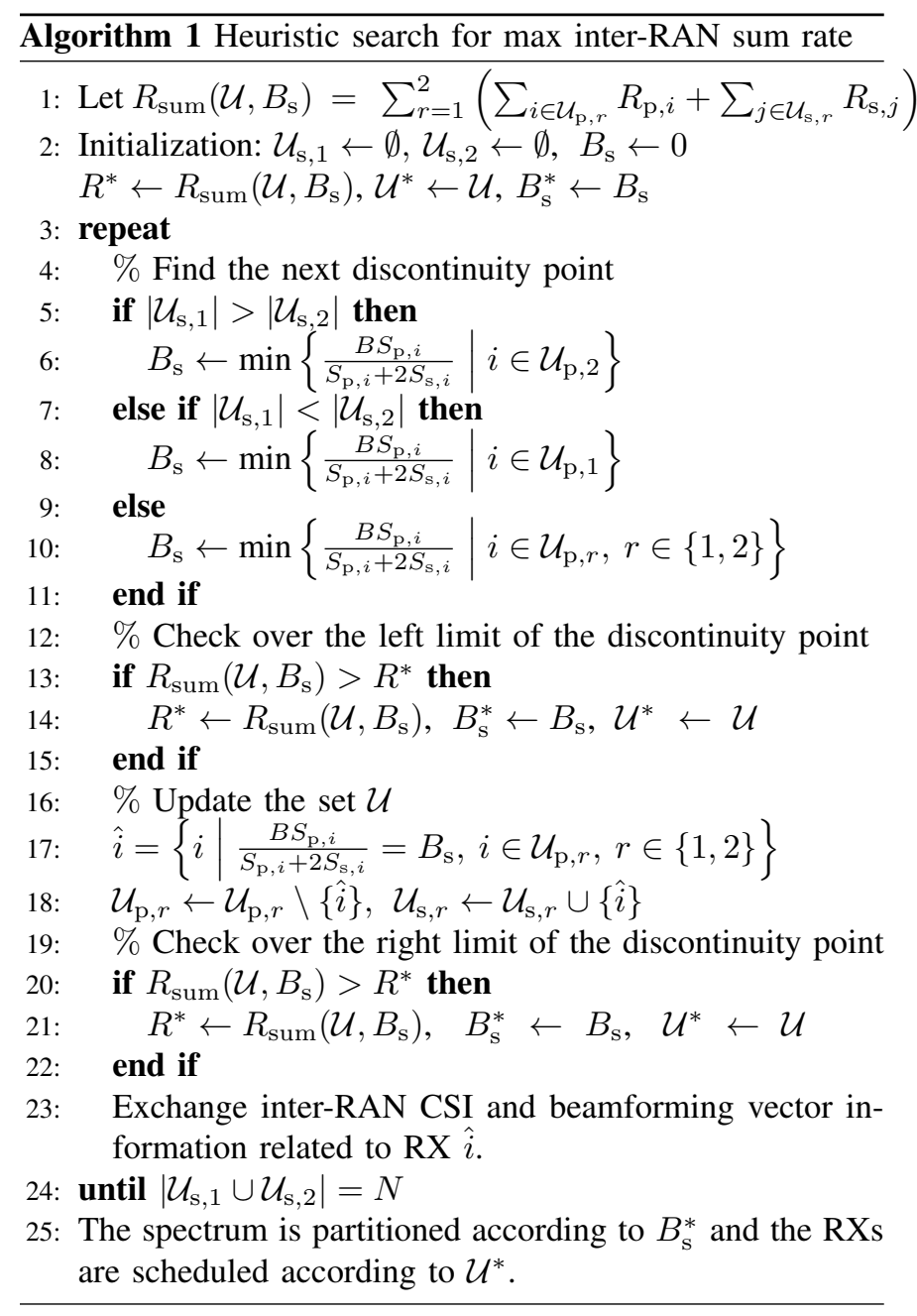

the frequency sub-band where it could achieve higher rate. Therefore, with $B_{\mathrm{s}}=0$, all the RXs are scheduled in the private frequency sub-bands. As we increase $B_{\mathrm{s}}$, the first discontinuity point occurs at $B_{\mathrm{s}}^{*}$, where

$$
B_{\mathrm{s}}^{*}=\min \left\{\frac{B S_{\mathrm{p}, i}}{S_{\mathrm{p}, i}+2 S_{\mathrm{s}, i}} \mid r \in\{1,2\}, i \in \mathcal{U}_{\mathrm{p}, r}\right\} .
$$

In order to find the next discontinuity point, the tentative set $\mathcal{U}$ is updated as $\mathcal{U}_{\mathrm{p}, r} \leftarrow \mathcal{U}_{\mathrm{p}, r} \backslash\left\{i^{*}\right\}$ and $\mathcal{U}_{\mathrm{s}, r} \leftarrow \mathcal{U}_{\mathrm{s}, r} \cup\left\{i^{*}\right\}$, where $\mathrm{RX} i^{*}$ is the RX which gets higher estimated rate if scheduled in the shared frequency sub-band, $B_{\mathrm{s}} S_{\mathrm{s}, i}>B_{\mathrm{p}} S_{\mathrm{p}, i}$, for all $B_{\mathrm{s}}>B_{\mathrm{s}}^{*}$,

$$
i^{*}=\left\{i \mid \frac{B S_{\mathrm{p}, i}}{S_{\mathrm{p}, i}+2 S_{\mathrm{s}, i}}=B_{\mathrm{s}}^{*}, r \in\{1,2\}, i \in \mathcal{U}_{\mathrm{p}, r}\right\} .
$$

This process continues until all the discontinuity points are found, or alternatively, $\left|\mathcal{U}_{\mathrm{s}, 1} \cup \mathcal{U}_{\mathrm{s}, 2}\right|=N$. However, we limit the difference between the number of scheduled RXs in the shared frequency sub-band from the two RANs to be at most one, $\left|\mathcal{U}_{\mathrm{s}, 1}\right|-\left|\mathcal{U}_{\mathrm{s}, 2}\right| \leq 1$, so that an operator do not over-utilize the shared resource. After the search is over, the spectrum partition $B_{\mathrm{s}}$ and the user grouping $\mathcal{U}$ which lead to the maximum inter-RAN sum rate are used to partition the

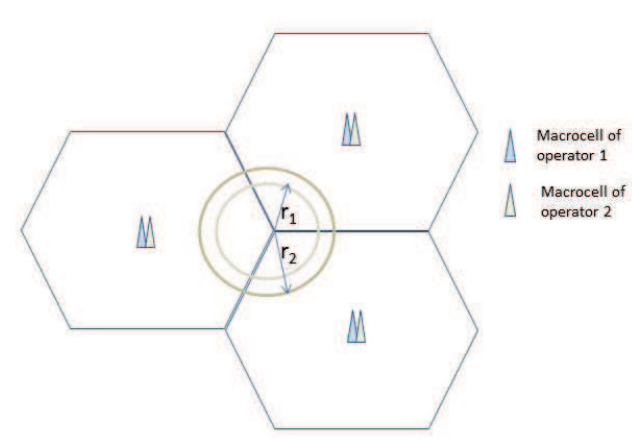

Fig. 1. Illustration of deployment scenario: Four RRHs and four UEs of RAN $r \in\{1,2\}$ are randomly distributed within radius of $r_{1}=50 \mathrm{~m}$ and $r_{2}=70 \mathrm{~m}$ of the hotspot area with $d_{\min , \mathrm{RRH}-\mathrm{RRH}}=10 \mathrm{~m}$ and $d_{\text {min, } \mathrm{UE}-\mathrm{RRH}}=3 \mathrm{~m}$ according to [12]. There is no minimum RRH to $\mathrm{RRH}$ and RRH to UE distance limit if the two RRHs or the RRH and the UE belong to different operators. The macrocells are introduced to generate external interference.

spectrum and schedule the RXs in the frequency sub-bands, as summarized in Algorithm 1. In the algorithm, we assume the RANs exchange intra-RAN sum rate information to calculate the inter-RAN sum rate.

\section{SIMULATION RESULTS AND ANALYSIS}

In this section, the performance of the proposed adaptive spectrum sharing scheme is analyzed under LTE heterogeneous network scenario consisting of macrocells and outdoor Remote Radio Heads (RRHs).

\section{A. Simulation Setup}

Two RANs composed of 4 RRHs and 4 UEs are considered. The two RANs cover an overlapping hotspot area located at the boundary of three macrocell sites, see Fig. 1. The two RANs are assumed to be connected using fiber optics to exchange signalling and channel information. Spectrum is only shared between the part of the two RANs consisting of the RRHs. The macrocells are included to study the effect of external interference on the spectrum sharing performance. Macrocells that belong to different operators are, assumed to share the same site. Within the considered hotspot area, the RRHs that belong to the same RAN are deployed according guidelines of [12] for co-channel hotspot deployment. The RRHs of the same RAN are uniformly distributed within a radius $r_{1}=50 \mathrm{~m}$ with a minimum RRH to RRH distance of $d_{\min , \mathrm{RRH}-\mathrm{RRH}}=$ $10 \mathrm{~m}$. The User Equipments (UEs) belonging to the same RAN are uniformly distributed within a radius of $r_{1}=70 \mathrm{~m}$ with a minimum UE to RRH distance of $d_{\min , \mathrm{UE}-\mathrm{RRH}}=3 \mathrm{~m}$. There is no minimum RRH to RRH and RRH to UE distance limit if the two RRHs or the RRH and the UE belong to different operators. The RANs are assumed to jointly serve their UEs with an equal per-stream power constraint of $P=$ $30 \mathrm{dBm}$ in their whole licensed spectrum, which is assumed to be $10 \mathrm{MHz}$. The total available spectrum for both RANs, thus, becomes $B=20 \mathrm{MHz}$. When the RANs are sharing their spectrum, the per-stream power constraint in the private and shared frequency sub-bands is scaled such that the power 


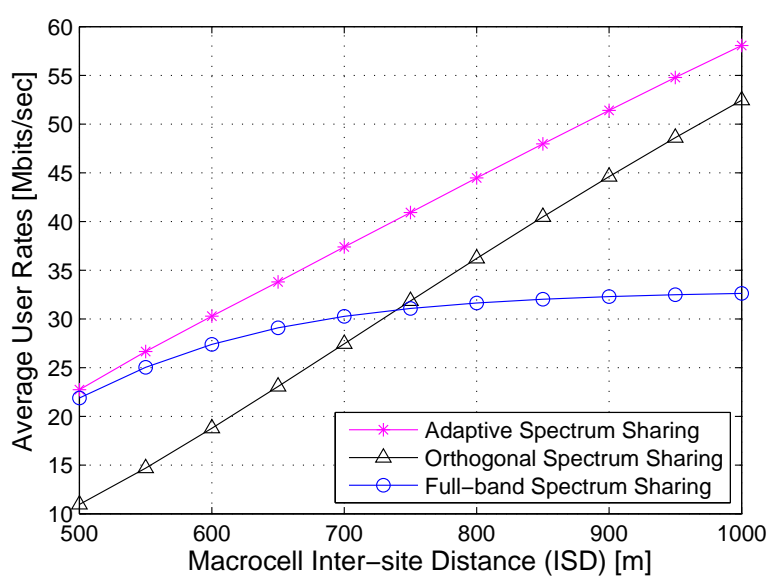

Fig. 2. Average user rates as a function of the macrocell intersite distance for the proposed and baseline spectrum sharing strategies.

spectrum density will remain the same across all sub-bands. The noise power and the incurred interference are also scaled according to the bandwidth.

The channel is assumed to include only the distance dependent path loss and the fast fading component. We use the path loss model for macrocell plus outdoor RRH/hotspot heterogeneous network (Case 1) according to [13], where the pathloss between a macro base station and a UE is given by

$$
P L_{\mathrm{Maco}-\mathrm{UE}}=128.1+37.6 \log _{10}(R), \quad R \text { in } \mathrm{km}
$$

and the pathloss between an RRH and a UE by

$$
P L_{\mathrm{RRH}-\mathrm{UE}}=140.7+36.7 \log _{10}(R), \quad R \text { in } \mathrm{km} .
$$

The fast fading component is modelled to be flat Rayleigh distributed. The macrocells use a transmission power of $46 \mathrm{dBm}$ in each carrier of $10 \mathrm{MHz}$. Other parameters are set according to the standard procedure for LTE simulation [13].

\section{B. Simulation Results}

The simulation results are obtained by averaging the UE rates over 20 RAN realizations for each macrosite intersite distance. In each RAN realization, we generate 50 user realizations each consisting of 20 fast fading realizations. As a baseline for comparison to the adaptive spectrum sharing, simulations for orthogonal and full-band non-orthogonal spectrum sharing approaches are also included. In orthogonal spectrum sharing, the two RANs use two orthogonal $10 \mathrm{MHz}$ frequency sub-bands, i.e. $B_{\mathrm{s}}=0$. In the full-band spectrum sharing, both RANs simultaneously use the whole $20 \mathrm{MHz}$ spectrum, i.e. $B_{\mathrm{s}}=B=20 \mathrm{MHz}$.

According to the simulation results, see Fig 2, the proposed adaptive spectrum sharing scheme outperforms both orthogonal and full-band spectrum sharing approaches under any external interference strength. The UE average rate with adaptive spectrum sharing is significantly higher than the average rate when orthogonal spectrum sharing is applied. The gain mainly comes from reusing part of the spectrum by the UEs served in the shared frequency sub-band, and from improving the received signal quality of the UEs served in the private frequency sub-band. The later is due to the reason that as some of the UEs are served in the shared frequency sub-band, the number of jointly served UEs in the private frequency sub-band decreases and thus improving their received signal quality. On the other hand, full-band spectrum sharing has better performance than orthogonal spectrum sharing under high external interference. However, it becomes inter-RAN interference limited as the external interference decreases.

\section{CONCLUSIONS}

In this work, we proposed an adaptive co-primary shared access scheme, where the spectrum is adaptively partitioned into private and non-orthogonally shared parts. A significant gain is obtained when the proposed scheme is used, especially in case of high and moderate external interference. This is typical in a scenario where there is local cooperation of clusters of base stations of different operators. Unlike with fullband spectrum sharing, the main advantage of the proposed adaptive spectrum sharing is that the users with controllable inter-operator interference could enjoy frequency reuse gain, while users facing strong inter-operator interference could still get improved received signal quality in the private frequency sub-band.

\section{ACKNOWLEDGMENT}

This work has been supported in part by the Academy of Finland under grant 254299.

\section{REFERENCES}

[1] Nokia Siemens Networks, "Wake-up call: Industry collaboration needed to make Beyond 4G networks carry 1000 times more traffic by 2020 ,' White Paper, Aug. 2011.

[2] International Telecommunication Union, "Estimated spectrum bandwidth requirements for the future development of IMT-2000 and IMTAdvanced," Report ITU-R M.2078, 2006.

[3] E. G. Larson and E. A. Jorswieck, "Competition versus cooperation on the MISO interference channel," IEEE J. Sel. Areas Commun., vol. 26, n. 7, Sep. 2008.

[4] L. Anchora, et al., "A Characterization of Resource Allocation in LTE Systems Aimed at Game Theoretical Approaches,' IEEE CAMAD, Dec., 2010.

[5] Jian Luo, et al., "Transmit Beamforming for Inter-Operator Spectrum Sharing: From Theory to Practice," IEEE ISWCS, Aug. 2012.

[6] Bennis M, Lasaulce S, Debbah M, "Inter-operator spectrum sharing from a game theoretical perspective," EURASIP J. Advances in Signal Processing, vol. 2009, Sep. 2009.

[7] J. Lindblom and E. Larsson, "Does Non-Orthogonal Spectrum Sharing in the same Cell Improve the Sum-Rate of Wireless Operators?", IEEE SPAWC, Jun. 2012.

[8] Gangula, Rajeev, et al., "On the Value of Spectrum Sharing among Operators in Multicell Networks.” IEEE VTC, Jun. 2013.

[9] Hans D. Schotten, Mikko A. Uusitalo, et al., "Intermediate description of the spectrum needs and usage principles," METIS 2020 Project Deliverable D5.1, vol. 1.0, Aug. 2013.

[10] P. de Kerret and D. Gesbert, "Sparse Precoding in Multicell MIMO Systems," IEEE WCNC, Apr. 2012.

[11] M. Grote and T. Huckle, "Parallel Preconditioning with Sparse Approximate Inverse," SIAM J. Scientific Computing, vol. 18, no. 3, May 1997.

[12] 3GPP TSG RAN WG1, "Evaluation assumptions for small cell enhancements - physical layer,' Tdoc R1-130856, Feb. 2013.

[13] 3GPP, "Further advancements for E-UTRA physical layer aspects (Release 9)," TR 36.814, V9.0.0 (2010-03), 2010. 